\title{
Torsion of the Gallbladder
}

\author{
Elizabeth A. Boonstra • Boudewijn van Etten • \\ Ted R. Prins • Egbert Sieders • Barbara L. van Leeuwen
}

Received: 23 August 2011 / Accepted: 20 September 2011 /Published online: 8 October 2011

(C) 2011 The Author(s). This article is published with open access at Springerlink.com

\begin{abstract}
Introduction A 77-year-old woman was seen with progressive abdominal pain.

Cases A CT scan was made and showed a large gallbladder extending into the right lower abdomen. Ultrasound was performed but demonstrated no gallstones. Laparoscopy showed a tordated, necrotic gallbladder that was attached to the liver only by the cystic artery and cystic duct. Cholecystectomy was performed.

Conclusions Torsion of the gallbladder is a rare but clinically important condition in which the diagnosis seldom is made preoperatively. In radiological and clinical signs of cholecystitis without gallstones, this condition should be considered.
\end{abstract}

\section{Keywords Gallbladder · Torsion · Cholecystitis · CT}

\section{Introduction}

Torsion of the gallbladder is a rare condition and an indication for urgent cholecystectomy. The diagnosis is seldom made before surgery. We will present a case of a patient with a tordated gallbladder and an overview of the literature.

E. A. Boonstra $(\bowtie) \cdot$ B. van Etten $\cdot$ B. L. van Leeuwen Department of Abdominal Surgery, University Medical Centre Groningen, Hanzeplein 1,

9713 GZ Groningen, The Netherlands

e-mail: e.a.boonstra@chir.umcg.nl

\section{T. R. Prins}

Department of Radiology, University Medical Centre Groningen, Groningen, The Netherlands

\section{E. Sieders}

Department of Hepatobiliary Surgery and Liver Transplantation, University Medical Centre Groningen,

Groningen, The Netherlands

\section{Case Report}

A 77-year-old lady presented with a distended abdomen with a constant tenderness in the right lower abdomen which worsened with movements. There was no rebound tenderness, defense, or palpable masses. There was no fever, nausea, or vomiting. Her medical history included osteoporotic fractures of T-7, T-9, and T-12, and L-1. She used pain-killers, medication indicated for osteoporosis, and acetylsalicyclic acid for unclear reasons. Laboratory investigation showed a hemoglobin of $7.2 \mathrm{mmol} / \mathrm{l}(7.5-9.9)$, a leukocyte count of $8.9 \times$ $10^{9} / 1\left(4-10 \times 10^{9} / \mathrm{l}\right)$, a CRP of $50 \mathrm{mg} / 1(<5 \mathrm{mg} / \mathrm{l})$, and a sodium of $127 \mathrm{mmol} / \mathrm{l}(135-145 \mathrm{mmol} / \mathrm{l})$. Liver tests and bilirubin were normal. With the differential diagnosis of a colon tumor or acute appendicitis, she was admitted to the hospital. The next day she underwent a CT scan with oral and intravenous contrast agents. This showed fluid collection in the right side of the abdomen. The radiologist interpreted this as the gallbladder with an abnormal location and configuration. The appendix was normal. Around the gallbladder, there was fat induration and induration of the right abdominal wall (Fig. 1). For more certainty about the fluid collection, an ultrasound investigation was performed. This revealed that the fluid collection indeed was the gallbladder in which the cystic duct could be followed until the common bile duct. The gallbladder wall was thickened and layered. Gallstones 


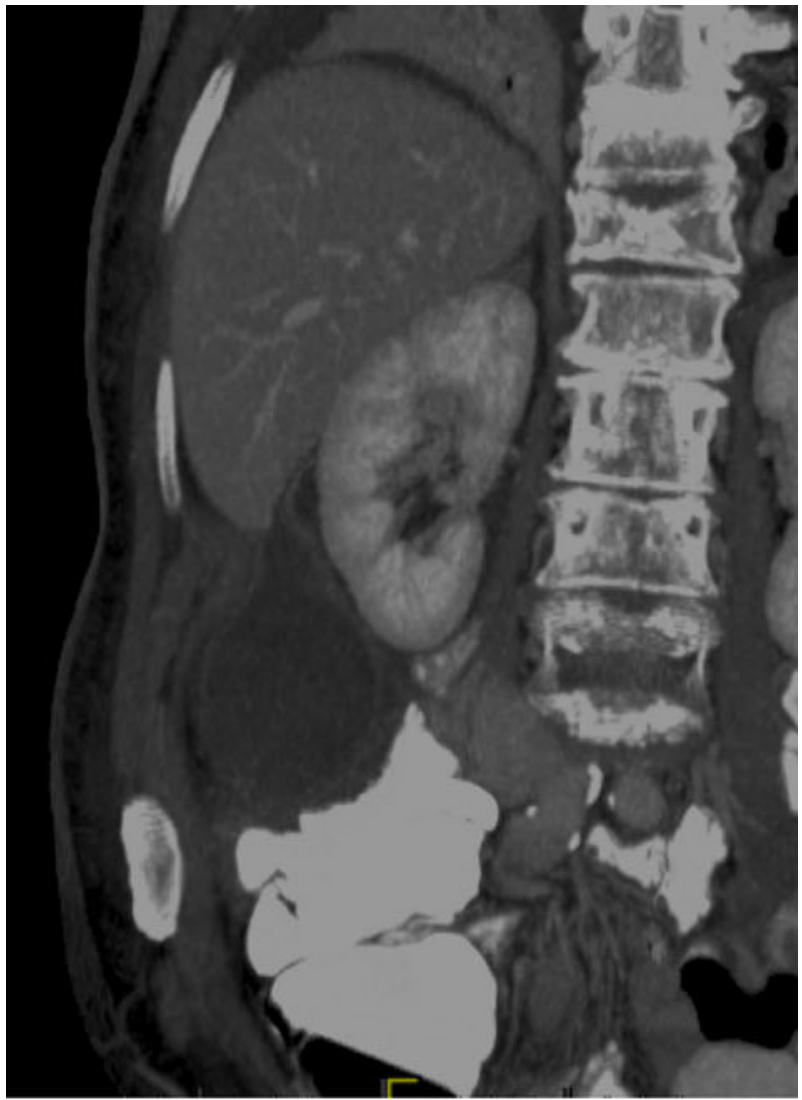

were not seen. The diagnosis of an acalculous cholecystitis was made, and a laparoscopic cholecystectomy was considered necessary. At laparoscopy, a gangrenous and much distended gallbladder was seen. The gallbladder was situated at the right side of the colon and reached the right lower quadrant of the abdomen. It was attached to the liver only by the cystic duct and artery. There was a $360^{\circ}$ torsion around the cystic duct and artery (Fig. 2). Because of the location of the gallbladder, distended bowel, and the kyphoscoliosis of the patient, an open cholecystectomy was performed. When the gallbladder was opened after surgery, there were no gallstones. The postoperative course was uncomplicated. Histopathologic investigation of the gallbladder showed an acute cholecystitis with hematoma and necrosis of the gallbladder wall.

\section{Overview of the Literature}

Torsion of the gallbladder is a rare cause of cholecystitis. The incidence is estimated at 1 in every 365.520 hospital
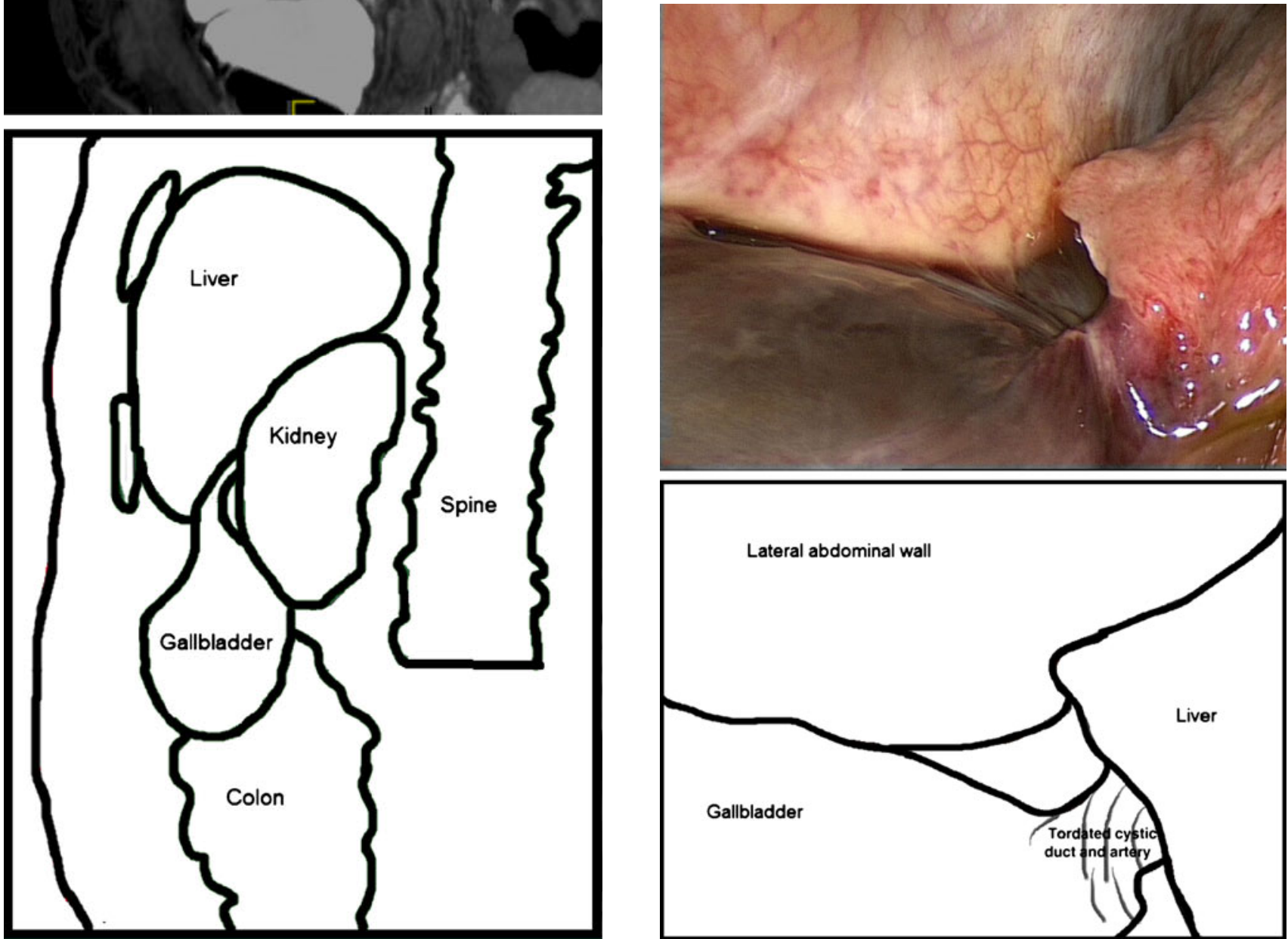

Fig. 1 CT image with the free-floating gallbladder

Fig. 2 The tordated cystic duct 
admissions. ${ }^{1}$ The first case was described by Wendel in $1898 .^{2}$ It is a condition mainly seen in elderly women, and the incidence appears to increase with increased life expectancy. ${ }^{3}$ Torsion of the gallbladder can only occur in patients with anatomic variation of gallbladder fixation to the liver. This could be a complete, but too long and wide mesentery or an incomplete mesentery covering only the cystic duct and artery. In these anatomic variations, there is a free-floating gallbladder. Another possibility is that relaxation and atrophy of a previously normal mesentery in the elderly cause visceroptosis. ${ }^{4,5}$ For the final torsion, a provocative moment is needed. In literature, kyphoscoliosis, forceful peristaltic movements, adhesions, atherosclerosis of the cystic artery, and sigmoid volvulus are reported as possible provocative factors. ${ }^{6}$ Striking is that the patient in our case report had a kyphoscoliosis. Symptoms of gallbladder torsion are acute onset of abdominal pain, nausea, and vomiting. These symptoms can be intermitting in case of $180^{\circ}$ torsion. $^{6}$ The often unusual location of a tordated gallbladder hampers making the right diagnosis and seldom is the diagnosis made before surgery. There are radiological signs that can indicate torsion of the gallbladder. Especially the presence of the gallbladder outside the normal anatomic fossa and a stretched cystic duct and gallbladder neck can indicate a free-floating gallbladder and are thereby risk factors for torsion of the gallbladder. Likewise, the absence of bile stones in a gallbladder with signs of cholecystitis can suggest a torsion of the gallbladder since an acalculous cholecystitis is very rare in otherwise healthy patients. A hypo-echogenic zone between the mucosa and serosa of the gallbladder is a sign of venous stasis and hematoma in the gallbladder wall. ${ }^{7}$ Besides an abnormal location of the gallbladder, a swirl sign of the cystic duct can be seen on $\mathrm{CT}$ images. The gallbladder will be more distended in torsion than in a normal acute cholecystitis. ${ }^{8}$ Treatment of a tordated gallbladder is acute (laparoscopic) cholecystectomy.
In conclusion, torsion of the gallbladder is a rare but clinical important condition in which the diagnosis is seldom made before surgery. At radiologic and clinical signs of acute cholecystitis in the absence of bile stones but with a free-floating gallbladder, this diagnosis must be considered.

Open Access This article is distributed under the terms of the Creative Commons Attribution Noncommercial License which permits any noncommercial use, distribution, and reproduction in any medium, provided the original author(s) and source are credited.

\section{References}

1. Yeh HC, Weiss MF, Gerson CD. Torsion of the gallbladder: the ultrasonographic features. J Clin Ultrasound 1989 Feb;17 (2):123-5.

2. Wendel AV. VI. A Case of Floating Gall-Bladder and Kidney complicated by Cholelithiasis, with Perforation of the Gall-Bladder. Ann Surg 1898 Feb;27(2):199-202.

3. Nakao A, Matsuda T, Funabiki S, Mori T, Koguchi K, Iwado T, et al. Gallbladder torsion: case report and review of 245 cases reported in the Japanese literature. J Hepatobiliary Pancreat Surg 1999;6(4):418-21.

4. Lemonick DM, Garvin R, Semins H. Torsion of the gallbladder: a rare cause of acute cholecystitis. J Emerg Med 2006 May;30 (4):397-401.

5. Gupta V, Singh V, Sewkani A, Purohit D, Varshney R, Varshney S. Torsion of gall bladder, a rare entity: a case report and review article. Cases J 2009;2:193.

6. Janakan G, Ayantunde AA, Hoque H. Acute gallbladder torsion: an unexpected intraoperative finding. World J Emerg Surg 2008;3:9.

7. Cameron EW, Beale TJ, Pearson RH. Case report: torsion of the gallbladder on ultrasound - differentiation from acalculous cholecystitis. Clin Radiol 1993 Apr;47(4):285-6.

8. Merine D, Meziane M, Fishman EK. CT diagnosis of gallbladder torsion. J Comput Assist Tomogr 1987 Jul;11(4):712-3. 